\title{
INFLUÊNCIA DO EXERCÍCIO FÍSICO NA SINDROME DA DOR PATELOFEMORAL
}

\author{
Douglas de Oliveira Chaves, Everton Alex Carvalho Zanuto, Robson Chacon Castoldi \\ Universidade do Oeste Paulista - UNOESTE, Curso de Educação Física, Presidente Prudente, SP.
}

\section{RESUMO}

Este trabalho de conclusão de curso visa pesquisar a síndrome da dor patelofemoral que é caracterizada pela dor na face anterior do joelho. Essa dor é resultado de uma compressão anormal da articulação do joelho, sendo assim, uma das desordens dolorosas mais comuns que o acomete. Quando analisado o movimento de flexão e extensão do joelho, observa-se que, a patela desliza sobre a face patelar do fêmur em uma espécie de trilho e o que à mantem sobre esse trilho são os músculos esqueléticos. Nota-se que, nas academias de musculação são cada vez mais frequente pessoas com dores no joelho, sejam elas atletas ou não. Diante disso, o objetivo geral deste trabalho é apresentar se há estudos científicos recentes referente a modulação de exercícios físicos em indivíduos com síndrome de dor patelofemoral, dessa forma, contribuirá para uma qualidade de vida aos mesmos. A metodologia utilizada trata-se de uma revisão sistemática de literatura com a utilização das bases de dados eletrônicos: Ovid, Bireme, Scielo e Medline. Pode-se afirmar que, em todas as bases de dados foram lançados os termos individualmente, posteriormente analisado e realizado o cruzamento das palavras. Dessa maneira, foram selecionados 10 artigos para a presente revisão. Os protocolos de reabilitação utilizados focaram no fortalecimento dos músculos responsáveis pela estabilização do quadril, em segundo lugar em quadríceps e isquiotibiais. Através da revisão realizada constata-se que, exercícios físicos sejam eles em treinamento resistido, alongamento e liberações miofasciais, quando modulado para indivíduos com síndrome de dor patelofemoral, promove melhora na função, qualidade de vida, redução da dor e estresse articular.

Palavras-chave: Patellofemoral pain, exercise e resistance training.

\section{ABSTRACT}

This course completion work aims to research the patellofemoral pain syndrome which is characterized by pain in the anterior face of the knee. This pain is a result of an abnormal compression of the knee joint, thus being one of the most common painful disorders that affects it. When analyzed the movement of knee flexion and extension, it is observed that, the patella slides on the patellar face of the femur in a kind of rail and what keeps it on this path are the skeletal muscles. It is noticed that, in the gymnasiums of bodybuilding are more and more frequent people with pains in the knee, be they athletes or not. Therefore, the general objective of this study is to present if there are recent scientific studies regarding the modulation of physical exercises in individuals with patellofemoral pain syndrome, in this way, it will contribute to a quality of life to them. The methodology used is a systematic literature review with the use of electronic databases: Ovid, Bireme, Scielo and Medline. It can be affirmed that in all the databases the terms were individually launched, later analyzed and realized the intersection of the words. Thus, 10 articles were selected for the present review. The rehabilitation protocols used focused on strengthening the muscles responsible for stabilizing the hip, secondly on the quadriceps and hamstrings. Through the review carried out, it can be observed that physical exercises are in resistance training, stretching and myofascial releases, when modulated for individuals with patellofemoral pain syndrome, it promotes improvement in function, quality of life, pain reduction and joint stress.

Keywords: Patellofemoral pain, exercise and resistance training. 


\section{INTRODUÇÃO}

A síndrome da dor patelofemoral é um termo utilizado para descrever a dor na face da anterior do joelho (MAIA et. al, 2011). Essa dor é resultado de uma compressão anormal da articulação do joelho. Quando analisamos o movimento de flexão e extensão do joelho, observamos que a patela desliza sobre o fêmur em uma espécie de trilho a tróclea femoral. O que a controla sobre a tróclea femoral são as musculaturas da coxa, que por sua vez, são responsáveis pela estabilização da patela, para que assim ela sempre esteja em sua função natural.

A dor na face anterior do joelho é o estágio inicial de uma patologia, onde não havendo uma intervenção evolui gradativamente assim para à condromalacia, que possui quatro estagio, sendo que o quarto estágio o mais grave é chamado de condropatia.

A condromalácia é classificada quanto ao grau de dor e degeneração da cartilagem como: grau 0 - cartilagem normal; grau I - área de hiposinal cartilaginoso; grau II - grau I e alterações do contorno; grau III - aspecto serrilhado com áreas de redução do sinal e grandes irregularidades do contorno; grau IV - úlceras cartilaginosas e alterações do osso subcondral. (SOUZA et al. 2008).

A condromalácia patelar é uma patologia que se caracteriza pelo amolecimento da cartilagem articular em decorrência do desequilíbrio bioquímico do líquido sinovial. Atividades aquáticas e exercícios de musculação são recomendados para reforçar a musculatura do quadríceps e melhorar a articulação do joelho (SOUZA et al. 2008). Hoje sabemos que as lesões na cartilagem do joelho são muito comuns, não só em indivíduos praticantes de esporte, como em indivíduos sedentários. Esportes de alto volume, principalmente corrida de fundo, estão geralmente bastante relacionados com a incidência de lesões condrais.

Belchior et al. (2006) e Cabral et al. (2003) verificaram que essa patologia incorpora $25 \%$ das lesões do joelho e $5 \%$ das lesões esportivas, acometendo principalmente jovens do sexo feminino na faixa etária de 15 a 25 anos.

As cartilagens têm como função evitar que as extremidades ósseas se toquem, aliviando forças de atrito durante o movimento. A degeneração da cartilagem pode ocorrer por prédisposição genética, incongruências, por incompatibilidade entre a aplicação de sobrecarga e capacidade de recuperação, subluxações sejam elas traumáticas ou por falta de força, desequilíbrio muscular e por repetido esforço irregular, excesso de movimento.

Partindo de uma das principais funções da musculatura esquelética que é a estabilização da estrutura corporal, musculaturas encurtadas ou enfraquecidas passam a causar desequilíbrio muscular. Sendo assim o exercício físico passa a ser um grande aliado no tratamento e prevenção dessa patologia. $O$ treino de força, também definido como treinamento contra resistência ou exercício resistido, e utilizado como uma terapia de reabilitação, na recuperação e ganho de força, aumento da massa muscular e das aptidões físicas, e os resultados são adquiridos tanto por estímulos mecânicos como metabólicos (LIMA et al, 2006).

Nas academias de musculação cada vez mais chegam pessoas com dores no joelho, sejam elas atletas ou não. A ideia é adquirir informações suficientes para poder atuar de forma qualificada para que assim possamos ser mais independentes para trabalhar de forma que melhore a qualidade de vida dos indivíduos. Desta forma o objetivo do presente estudo foi analisar o efeito de diferentes tipos de exercício na síndrome da dor patelofemoral em adultos.

\section{MÉTODO}

Foi realizada uma busca sistemática na literatura utilizando as bases de dados; Ovid, Bireme, SCIELO e MEDLINE, a partir dos descritores: resistence training, exercise e patellofemoral pain, onde o critério de seleção foi à data de publicação de 2006-2017 e artigos originais.

$\mathrm{Na}$ base de dados Ovid após o cruzamento das palavras patellofemoral pain e resistence training e exercise e patellofemoral pain, encontrou-se 33 artigos. 
Na base de dados Bireme após o cruzamento das palavras patellofemoral pain e resistence training e exercise e patellofemoral pain, encontrou-se 82 artigos.

$\mathrm{Na}$ base de dados SCIELO após o cruzamento das palavras patellofemoral pain e resistence training e exercise e patellofemoral pain, encontrou-se 11 artigos.

$\mathrm{Na}$ base de dados MEDLINE após o cruzamento das palavras patellofemoral pain e resistence training e exercise e patellofemoral pain, encontrou-se 91 artigos.

\section{RESULTADOS}

Em todas as bases de dados foram lançados os termos individualmente e posteriormente realizou-se o cruzamento das palavras. Dentre os artigos encontrados o critério de seleção foi à data de publicação de 2006-2017, artigos originais, excluindo estudos de caso, revisões sistemáticas e posteriormente verificaram-se os títulos que constavam dados sobre o objeto de estudo em questão, em seguida os resumos foram lidos e selecionados os que mais descrevem sobre a relação do exercício e condromalacia, afunilando ainda mais sobre o tema abordado. Sendo assim, foram selecionados 10 artigos para a presente revisão. (FIGURA 1)

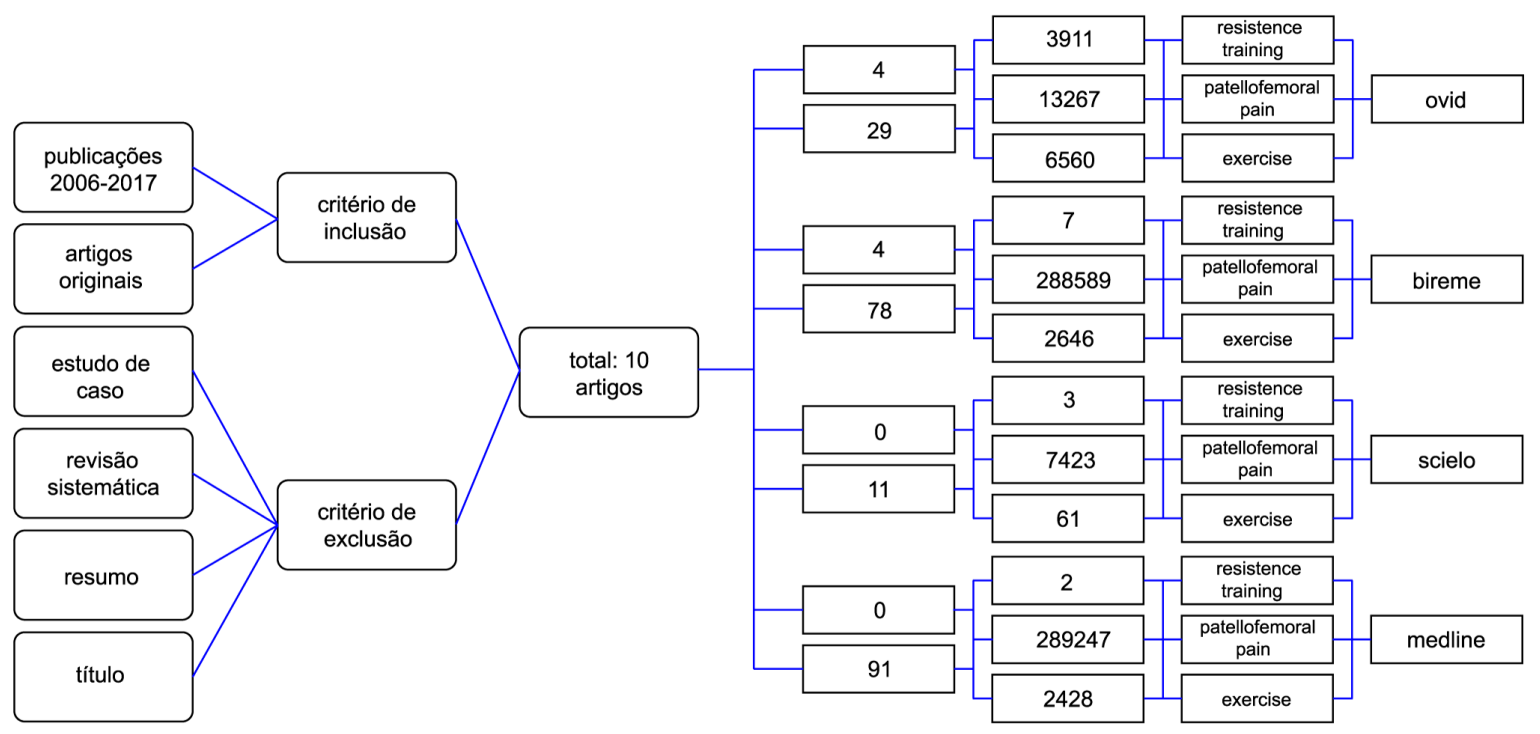

Figura 1. Organograma explicativo do método de seleção dos artigos. 


\begin{tabular}{|c|c|c|c|c|}
\hline & Autor & Amostra & Protocolo de treinamento & Conclusão \\
\hline 1 & $\begin{array}{l}\text { MIYAMOTO,G,C, et al. } \\
\text { (2010) }\end{array}$ & $\begin{array}{l}\text { Participaram do estudo } 12 \\
\text { voluntários com dor } \\
\text { patelofemoral, dominância de } \\
\text { membro inferior direito e idade } \\
\text { média de } 20 \text { anos. }\end{array}$ & $\begin{array}{l}\text { O tratamento que consistiu em alongamento } \\
\text { muscular segmentar bilateral dos músculos } \\
\text { isquiotibiais, tríceps sural e quadríceps femoral, } \\
\text { com duração de } 30 \text { segundos e } 10 \text { repetições para } \\
\text { cada músculo. }\end{array}$ & $\begin{array}{l}\text { O tratamento possibilitou melhoras nos } \\
\text { sintomas apresentados pelos pacientes } \\
\text { com dor patelofemoral, como } \\
\text { alinhamento, dor e função do joelho. } \\
\text { Todas as variáveis apresentaram } \\
\text { diferença significante após o } \\
\text { tratamento }(p<0,05) \text {. }\end{array}$ \\
\hline 2 & $\begin{array}{l}\text { TIGGELEN,D,V, et al. } \\
\text { (2009) }\end{array}$ & $\begin{array}{l}92 \text { cadetes masculinos saudáveis } \\
\text { do primeiro ano da Academia } \\
\text { Real Militar, com idades entre } 17 \\
\text { e } 27 \text { anos. }\end{array}$ & $\begin{array}{l}\text { O treinamento básico militar é um programa de } \\
\text { treinamento de } 6 \text { semanas, composto por corrida, } \\
\text { caminhadas com mochilas }(20-30 \mathrm{~kg}) \text { e exercícios } \\
\text { táticos militares. }\end{array}$ & $\begin{array}{l}\text { Dos restantes } 79 \text { indivíduos, } 26(32 \%) \\
\text { desenvolveram PFP durante } 6 \text { semanas } \\
\text { de treinamento extenuante. }\end{array}$ \\
\hline 3 & $\begin{array}{l}\text { MICHELLE C, et. al } \\
\text { (2006) }\end{array}$ & $\begin{array}{l}28 \text { indivíduos (14 controles, } 14 \\
\text { experimentais) entre Idade de } \\
18 \text { e } 42 \text { anos. Para o grupo } \\
\text { experimental, recrutamos } 5 \\
\text { homens e } 9 \text { mulheres. }\end{array}$ & $\begin{array}{l}\text { Programa de reabilitação de } 6 \text { semanas. Consistiu } \\
\text { de exercícios resistidos que focaram o } \\
\text { fortalecimento quadríceps e musculatura abdutora } \\
\text { de quadril. }\end{array}$ & $\begin{array}{l}\text { Os indivíduos diagnosticados com dor } \\
\text { patelofemoral, responderam } \\
\text { favoravelmente para um programa de } \\
\text { exercícios terapêuticos que quadríceps } \\
\text { incorporado e fortalecimento da } \\
\text { musculatura do quadril. }\end{array}$ \\
\hline 5 & TELLES, D et al. (2016) & $\begin{array}{l}18 \text { pacientes, de ambos os sexos } \\
\text { com idade de } 27 \text { a } 73 \text { anos. }\end{array}$ & $\begin{array}{l}\text { Um grupo } 1 \text { de } 9 \text { pacientes foi tratado com o } \\
\text { fortalecendo dos músculos do quadril e o grupo } 2 \\
\text { que foi tratado com fortalecimento dos músculos e } \\
\text { técnicas de liberação miofasciail e alongamento. }\end{array}$ & $\begin{array}{l}\text { O estudo mostrou que os participantes } \\
\text { no grupo } 2 \text { tiveram redução da } \\
\text { intensidade da dor e melhora do índice } \\
\text { de incapacidade da extremidade } \\
\text { inferior, A adição de técnicas } \\
\text { miofasciais deve ser considerada para } \\
\text { melhorar a funcionalidade dos } \\
\text { membros inferiores e reduzir a dor em } \\
\text { pacientes com dor anterior do joelho. }\end{array}$ \\
\hline 6 & KURIKI,H.U. (2009) & $\begin{array}{l}33 \text { voluntárias- } 11 \text { com } \\
\text { diagnóstico médico de } \text { dor } \\
\text { fêmoro-patelar e } \\
\text { clinicamente normais, }\end{array}$ & $\begin{array}{l}\text { Foram submetidas à avaliação por meio de } \\
\text { eletromiografia de superfície das porções medial e } \\
\text { lateral do quadríceps; análise tridimensional de } \\
\text { movimento, e plataforma de força durante a } \\
\text { subida de degraus. }\end{array}$ & $\begin{array}{l}\text { As voluntarias do grupo dor } \\
\text { patelofemoral foram caracterizadas por } \\
\text { um atraso no tempo de atividade } \\
\text { máxima do músculo vasto medial em } \\
\text { relação ao vasto lateral, sendo assim } \\
\text { mais propensos a desenvolver síndrome } \\
\text { da dor patelofemoral. }\end{array}$ \\
\hline
\end{tabular}




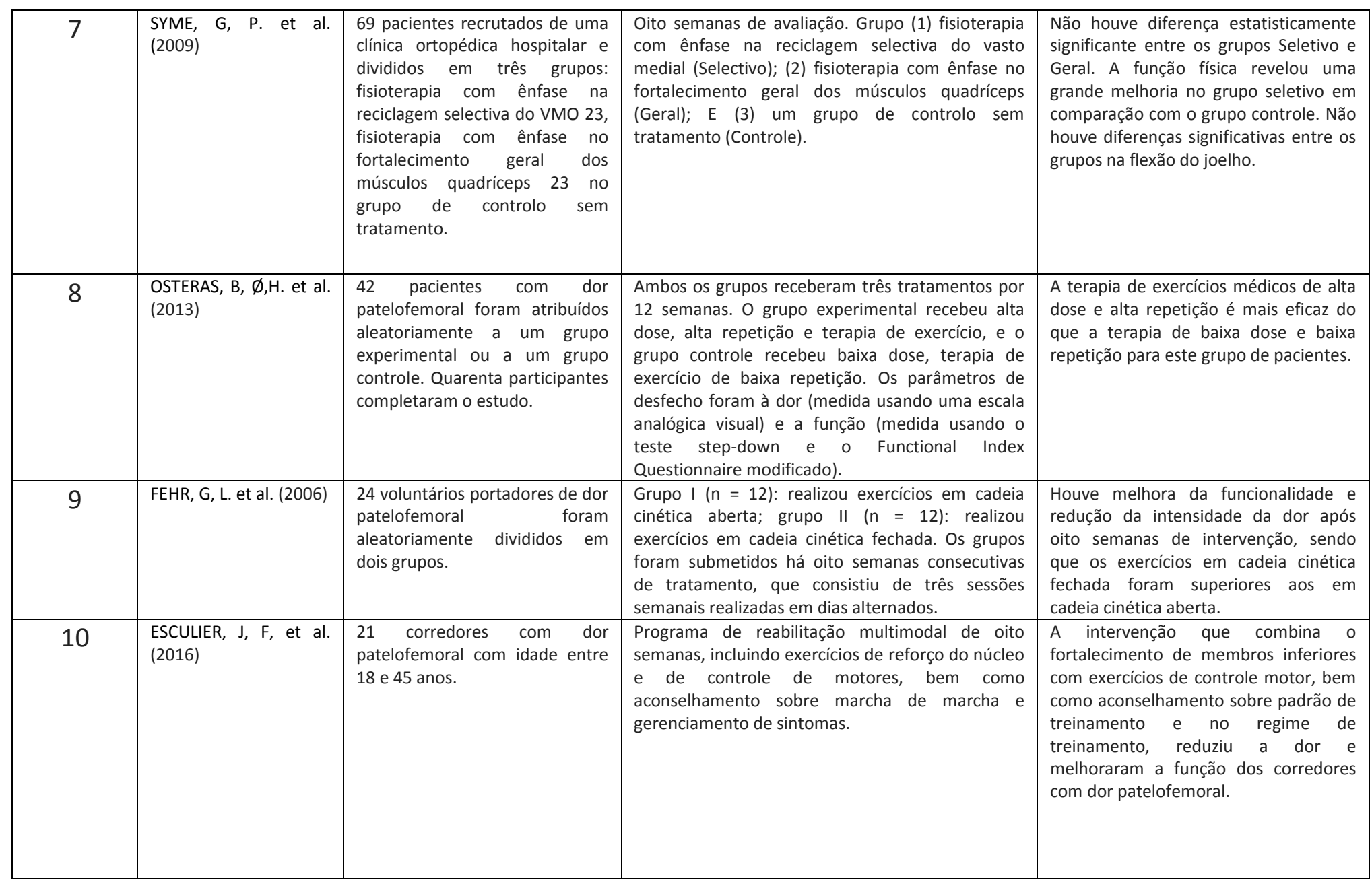




\section{DISCUSSÃO}

Em um estudo realizado por Miyamoto et al. (2010) avaliaram os efeitos do alongamento muscular segmentar. O tratamento constituindo-se de auto-alongamento de isquiotibiais, quadríceps femoral, e tríceps sural. Neste estudo observou-se uma melhora significativa do ângulo $\mathrm{Q}$ após o alongamento segmentar da musculatura. Dado a melhora do alinhamento patelar, devido alongamento da musculatura encurtada, tendo assim uma diminuição da dor e estresse articular. Telles et al. (2010) obtiveram resultados interessante, com redução da intensidade da dor na face anterior no joelho, onde além de alongamento segmentar realizou manobras de liberação miofascial e fortalecimento dos músculos do quadril. Os participantes do estudo foram orientados a repetirem os exercícios em casa sem resistência de elásticos ou tornozeleiras 5 vezes na semana. A técnica de liberação miofascial foi realizada no quadríceps femoral, no músculo tensor do fáscia lata e banda iliotibial. O alongamento seguiu o mesmo protocolo de (MIYAMOTO et al. 2010) em relação ao tempo de estiramento da musculatura. É interessante destacar que às adições técnicas miofasciais devem ser consideradas para melhorar a funcionalidade dos membros inferiores.

A liberação miofascial utilizada foi no conceito de liberação ou soltura. A técnica utilizada é realizada manualmente onde a aplicação apropriada de estresse resulta em relaxamento do musculo, onde a pretensão é recuperar a simetria da forma e função da musculatura (BHARANGHA IHNGRUDA, 2005).

A flexibilidade muscular é um componente da aptidão física muito importante e está relacionada à saúde e ao desempenho atlético, podendo ser definida como a habilidade para mover uma articulação através de uma amplitude de movimento normal sem estresse excessivo para a unidade músculo-tendínea (CHANDLER et al., 1990). Abdal-Junior (2011) define o alongamento como um exercício que promove o aumento ou manutenção da flexibilidade que envolve uma força de superar a resistência muscular. Durigon (1995), diz que ao alongarmos um músculo, interferimos com a circuitaria do reflexo miotático, que tem como função primária, entre outras, a proteção da estrutura muscular. Como Peeler et. al. (2007) menciona eu seu estudo que a posição articular patelofemoral, pode estar relacionado à diminuição da flexibilidade, promovendo assim um encurtamento muscular provocando um mau alinhamento patelar gerando assim dor na face anterior do joelho. Sendo assim a ideia é identificar o desequilíbrio muscular através de avaliações, para que assim seja feita a correção através do alongamento, equilibrando assim as forças de contração e aumentando a capacidade de contração muscular, diminuindo a tensão articular patelofemoral possivelmente ocasionada pelo desequilíbrio tensional da musculatura.

Tiggelen et al. (2009) realizaram estudo, onde avaliou através de um eletromiógrafo o atraso na atividade do vasto medial e vasto lateral contribui para o desenvolvimento da dor patelofemoral em homens previamente saldáveis. O estudo utilizou um treinamento de militar como protocolo de treinamento tendo um programa diário consiste em 12 a 15 horas de atividades programadas, com duração de 6 semanas, onde ao final do estudo 32\% dos indivíduos desenvolveram dor patelofemoral. O estudo demonstrou que embora a causa da dor patelofemoral seja multifatorial o atraso de atividade entre vasto medial e vasto lateral é significativamente relevante em desenvolver uma dor patelofemoral. Kuriki (2009) em estudo semelhante avaliaram tridimensional de movimento vasto medial e vasto lateral, onde constatou que as voluntarias do grupo SDFP foram caracterizadas por um atraso no tempo de atividade máxima do músculo vasto medial em relação ao vasto lateral.

Michelle et al. (2006) realizaram um programa de reabilitação com pacientes com síndrome da dor patelofemoral. O estudo teve duração de 6 semanas, onde os exercícios realizados focaram no fortalecimento do quadríceps e abdutores do quadril. 0 estudo mostrou que a diferença no tempo de atividade do vasto medial e lateral sofrem alterações após o 
programa de reabilitação, dado também encontrado em Tiggelen et al. (2009) Kuriki (2009). O programa de reabilitação mostrou-se eficaz na diminuição de dor e melhora funcional dos pacientes, entretanto, o autor sugere que futuras pesquisas realizem com um grupo populacional maior e avalie maiores dimensões. Mulheres com síndrome patelofemoral têm abdutores de quadril $26 \%$ mais fracos e rotadores externos de quadril $36 \%$ mais fracos, quando comparado com grupo controle saudável, (IRELAND, et. al. 2003). Brindle et. al. (2003) constataram em seu estudo atraso na ativação de glúteo médio contribui para síndrome da dor patelofemoral.

Syme et al. (2009) em seu estudo de 8 semanas compararam os efeitos da reabilitação do vasto medial com a reabilitação com ênfase no fortalecimento geral dos músculos do quadríceps femoral tendo como objetivo a redução da dor, melhora da funções e qualidade de vida dos pacientes com a síndrome da dor patelofemoral. Os pacientes foram divididos em três grupos; grupo 1 seletivo (vasto medial), grupo 2 (geral) e grupo 3 (controle). Ambos os grupos tratados apresentaram melhora estatística significativa e grandes em relação à melhora nas funções. Tanto quanto à abordagem seletiva, quanto à geral tiveram resultados aceitáveis, a reabilitação de pacientes com síndrome da dor patelofemoral.

Osteras et al. (2013) em seu estudo compararam doses de exercícios em dois grupos de pacientes com síndrome da dor patelofemoral. Ambos os grupos receberam o tratamento 3 vezes semanais durante 12 semanas. O grupo experimental recebeu altas cargas de exercício e altas repetições, e o grupo controle recebeu baixas cargas de exercício e baixas repetições. Observou-se que a terapia com exercícios de altas cargas e altas repetições é mais eficaz do que a terapia com doses baixa e de baixas repetições.

Esculier et al. (2016) avaliaram 21 corredores com idade entre 18 e 45 anos com síndrome da dor patelofemoral, durante 8 semanas em um programa de reabilitação que consistia em realizar exercícios que combinavam o fortalecimento de membros inferiores com exercícios de controle moto, conselhos sobre a execução de biomecânica e sobre a marcha. Dado os resultados da intervenção, observou-se redução na dor e melhora da funcional dos corredores com síndrome da dor patelofemoral.

Fehr et al. (2006) por sua vez, em seu estudo, analisaram os efeitos de exercícios terapêuticos de cadeia cinética fechada e exercícios de cadeia cinética aberta, no tratamento da síndrome da dor patelofemoral. Os voluntários foram divididos em grupo que realizaram exercícios de cadeia cinética aberta e grupo que realizaram exercícios de cadeia cinética fechada. O tratamento teve duração de 8 semanas, sendo ela realizadas em 3 sessões semanas realizadas em dias alternados. 0 estudo encontrou aumentos significativos na funcionalidade e redução da intensidade da dor em ambos os grupos tratados, entretanto o grupo que realizou exercícios de cadeia cinética fechada obteve resultados superiores.

Miao et al. (2015) investigaram o efeito do semisquat de dupla perna, com adução do quadril na ativação do vasto medial e vasto lateral (que seria um $1 / 3\left(60^{\circ}\right.$ ) de agachamento), em indivíduos com síndrome da dor patelofemoral. 0 estudo foi realizado com 30 indivíduos com síndrome da dor patelofemoral e 30 indivíduos saudáveis como grupo controle. O exercício de semisquat de dupla perna com adução de quadril, foi realizado com ajuda de uma faixas elásticas. o estudo observou aumento na atividade do vasto medial quando comparado o exercício semisquat com adução de quadril ao mesmo exercício sem adução de quadril. Sendo assim é uma ferramenta para equiparar equilibrando atividade muscular entre vasto medial e vasto lateral. 0 autor destaca limitações no estudo, dentre elas o grau de flexão do joelho de $60^{\circ}$, que poderia ter sido avaliado em uma variedade maior de ângulos. 


\section{CONSIDERAÇÕES FINAIS}

Através da revisão realizada constata-se que, exercícios físicos sejam eles em treinamento resistido, alongamento e liberações miofasciais, quando modulado para indivíduos com síndrome de dor patelofemoral, promove melhora na função, qualidade de vida, redução da dor e estresse articular. Observa-se que, há um consenso em trabalhar músculos estabilizadores do quadril, visando à distribuição das forças realizadas. Nota-se que, há um desequilíbrio muscular em indivíduos com síndrome de dor patelofemoral.

A questão de reestruturação da marcha foi citada em um dos estudos, exercícios de controle moto, conselhos sobre a execução de biomecânica, ou seja, educativo sobre a marcha e corrida. Dessa forma, os estabilizadores do quadril sendo fortalecidos, contribuirão numa melhora significativa na mecânica de movimentos.

É inegável que, pesquisas futuras que utilizem a combinação das técnicas de reabilitação, como o alongamento, fortalecimento de vasto medial e lateral, pensando no equilíbrio muscular, fortalecimento de abdutores, adutores e rotadores internos e externos de quadril, juntamente com alongamento de possível falta de flexibilidade, conciliada com liberação miofascial e exercícios didáticos de marcha.

\section{REFERÊNCIAS}

1. B. OSTERAS et al. Dose-response effects of medical exercise therapy in patients with patellofemoral pain syndrome: a randomized controlled clinical trial/ Physiotherapy 99 (2013) 126-131.

2. BELCHIOR, A.C.G., Arakaki, J.C., Efeitos na medida do ângulo Q com a contração isométrica voluntária máxima do músculo quadriciptal, Rev. Bras. Esporte, v.12, n.1, jan/fev 2006.

3. BRINDLE TJ, Mattacola CG, McCrory JL. Electromyographic changes in the gluteus medius during stair ascent and descent in subjects with anterior knee pain. Knee Surg Sports Traumatol Arthrosc 2003; 11:244-51, https://doi.org/10.1007/s00167-003-0353-z.

4. CABRAL, C.M. N, Melin, A.M.O., Neves, I.C. N, Marques, A.P., Fisioterapia em pacientes com Síndrome Femoropatelar: Comparação de exercícios em cadeia cinética aberta e fechada. Acta Ortop Bras. 2008, https://doi.org/10.1590/S1413-78522008000300012.

5. DURIGON, O.F.S. O alongamento muscular. Rev. Fisioter. Univ. São Paulo, 2 (1): 40-4, jan. / jul, 1995.

6. ESCULIER, J. F, Laurent J. Bouyer, and Jean-Sébastien Roy/ The Effects of a Multimodal Rehabilitation Program on Symptoms and Ground-Reaction Forces in Runners with Patellofemoral Pain Syndrome/ Journal of Sport Rehabilitation, Vol. 25, No. 1, 2016, https://doi.org/10.1123/jsr.2014-0245.

7. FEHR, F. G, Junior, A. C, Cacho, E. W. A e Miranda J. B / Efetividade dos exercícios em cadeia cinética aberta e cadeia cinética fechada no tratamento da síndrome da dor femoropatelar/ Rev Bras Med Esporte _ Vol. 12, № 2 - Mar/Abr, 2006.

8. FERREIRA. F. C.L. efeitos dos exercícios de musculação para o fortalecimento da musculatura da coxa em portadora de condromalácia patelar/. Coleção Pesquisa em Educação Física - Vol.7, no $3-2008$. 
9. IRELAND M, Willson J, Ballantyne B, Davis I. Hip strength in females with and without patellofemoral pain. J Orthop Sports Phys Ther 2003; 33:671-6, https://doi.org/10.2519/jospt.2003.33.11.671.

10. JUNIOR, A. A Teoria e Metodologia do Treinamento Esportivo; Futebol Alongamento e Flexibilidade (2011).

11. KURIKI, H.U. Análise de parâmetros biomecânicos relacionados à Síndrome Dolorosa Fêmoro-Patelar. Dissertação (Mestrado) - Programa de Pós-Graduação Inter unidades em Bioengenharia (EESC/FMRP/IQSC), Universidade de São Paulo, São Carlos, 2009.

12. MEIRA S. S; Vilela A. B. A; Souza; Matos I. M, Bezerra S; Pestana A M S, Síndrome da dor femoropatelar: uma revisão sistemática. Brazilian Journal of Biomechanics, v. 13, n. 25, 2012.

13. MIAO. P, Yi Xu, Cuihuan Pan, Hao Liu, e Chuhuai Wang. Vastus medialis oblique and vastus lateralis activity during a double-leg semisquat with or without hip adduction in patients with patellofemoral pain syndrome, Miao et al. BMC Musculoskeletal Disorders (2015) 16:289, https://doi.org/10.1186/s12891-015-0736-6.

14. MICHELLE C. Boling, MS, ATC, Lori A. Bolgla, PT, PhD, ATC, Carl G. Mattacola, PhD, ATC, Tim L. Uhl, PT, PhD, ATC, Robert G. Hosey, MD Outcomes of a Weight-Bearing Rehabilitation Program for Patients Diagnosed with Patellofemoral Pain Syndrome Arch Phys Med Rehabil Vol 87, November 2006.

15. MIYAMOTO G. C; Soriano F.R; Cabral C. M. N. Rev. Bras. Med. Esporte - Vol. 16, N $4-$ Jul/Ago, 2010.

16. NIJS. J, Geel CV, Auwera CV, Velde BV. Diagnostic value of five clinical tests in patellofemoral pain syndrome. Man Ther 2006; 11:69-77, https://doi.org/10.1016/j.math.2005.04.002.

17. OLIVEIRA, L. V, Saad, M. C, Felício, L. R, Grossi, D. B, Análise da força muscular dos estabilizadores do quadril e joelho em indivíduos com Síndrome da Dor Femoropatelar / Fisioter Pesq. 2014; 21(4): 327-332.

18. PEELER J, Anderson J E. Effectiveness of static quadriceps stretching in individuals with patellofemoral joint pain. Clin J Sport Med 2007; 17:234-41, https://doi.org/10.1097/JSM.0b013e3180f60afc.

19. RICHARD B. Souza, Christopher M. Powers. Differences in Hip Kinematics, Muscle Strength, and Muscle Activation Between Subjects with and Without Patellofemoral Pain/ journal of orthopaedic \& sports physical therapy | volume 39 | number 1 | january 2009 | 13.

20. SYME G. P. Rowe D. Martin G. Daly et al. / Disability in patients with chronic patellofemoral pain syndrome: A randomised controlled trial of $\mathrm{VMO}$ selective training versus general quadriceps strengthening/ Manual Therapy 14 (2009) $252 \mathrm{e} 263$.

21. TELLES, G. D. R. Cristovão; Belache F. A. T. C.; Santos M. R. A.; Almeida R. S.; Nogueira L. A. C. The effect of adding myofascial techniques to an exercise programme for patients with anterior 
knee pain - A randomized clinical trial Journal of Bodywork \& Movement Therapies (2016), doi: 10.1016/j.jbmt.2016.02.007, https://doi.org/10.1016/j.jbmt.2016.02.007.

22. TIGGELEN D. V, PT, Sallie Cowan, PT, PhD, Pascal Coorevits the American Journal of Sports Medicine, Vol. 37, No. 6 2009. DOI: 10.1177/0363546508331135, https://doi.org/10.1177/0363546508331135. 